\title{
The effect of human presence on the extinction performance of a cat'
}

John A. Corson MCGILL UNIVERSITY

\begin{abstract}
Abstraet
One cat was taught to turn a wheel for milk reinforcement. During extinction of this habit the cat made significantly more responses while $\mathrm{E}$ was in the experimental room than when $\mathrm{E}$ was out. Possible determinants and implications of this effect are discussed.

\section{Problem}

This report concerns a result which appeared unexpectedly during a study of the effect of adrenocorticotrophic hormone (ACTH) on learning and extinction in cats. There has been previous recognition of the occasional relationship of a subject's responses to changes in stimuli other than those originally thought to be important by the experimenter. The present investigation was concerned with the apparent influence of a factor which must be considered in future design and interpretation of a large body of research.
\end{abstract}

\section{Method}

The subject was one $2500 \mathrm{gm} 18$ month old cat obtained from the cat colony at McGill Biology Department. The cat was kept on a $22 \mathrm{hr}$. food and liquid deprivation schedule.

The apparatus was a 24 in square box in which a dipper of milk was automatically presented for $10 \mathrm{sec}$. following each $1 / 2$ rotation of a wheel. This delivery was accompanied by a noisy electric motor and the onset of a small light in the area of the liquid dipper. From inside the apparatus $\mathrm{S}$ could see $\mathrm{E}$ through the spokes of the wheel, through the small hole cut to allow the cat access to the liquid dipper and through a small plexiglass window in the roof over the liquid dipper area.

$\mathrm{S}$ was wormed and handled during the first two weeks in the laboratory. At the beginning of the third week the deprivation schedule was put into effect and $S$ was placed in the apparatus each day for $1 \mathrm{hr}$. of shaping. After 10 days $\mathrm{S}$ began to turn the wheel without assistance and did so $1 \mathrm{hr}$. per day for days 11 through 22 and then for $1 / 2 \mathrm{hr}$. per day from day 23 to the end of the experiment. Beginning on day $29 \mathrm{~S}$ was given daily intramuscular injections of $12.5 \mathrm{U}$. S. P. units of adrenocorticotrophic hormone (ACTH) in carboxymethylcellulose vehicle ${ }^{2}$. These injections were administered $15 \mathrm{~min}$. before each session. This ACTH preparation has been shown to be active for 2 to 3 days after a single injection. On day 32 extinction was begun and on day $42 \mathrm{ACTH}$ was discontinued. After a 4 day rest the animal was tested for 9 more days without the influence of ACTH.

On day 32 (first extinction day) $\mathrm{E}$ began keeping a record of the amount of time he was spending in the

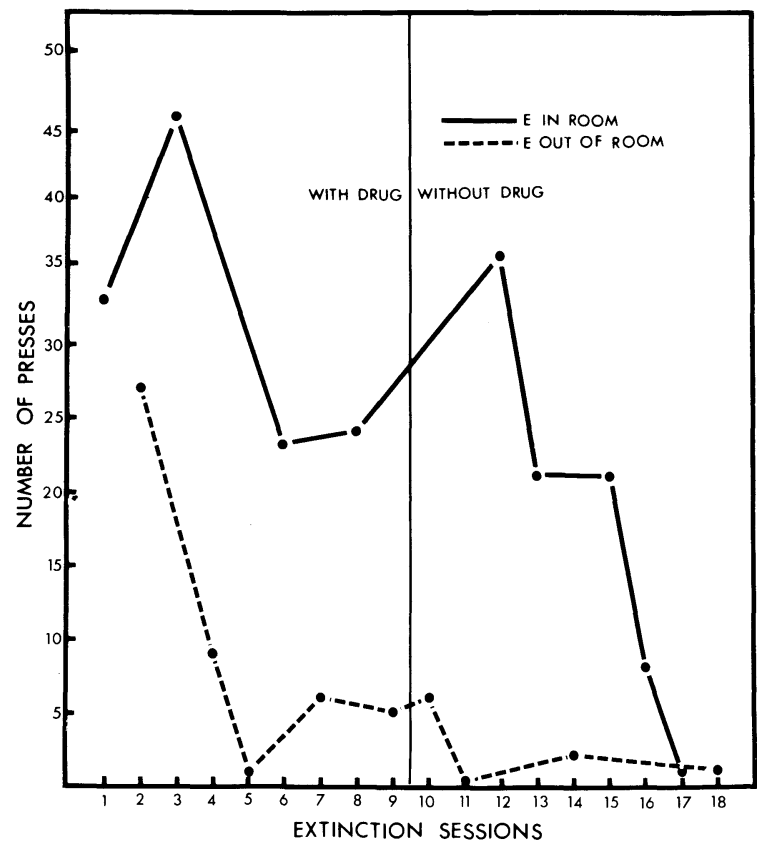

Fig. 1. Number of presses during each extinction session with the experimenter in (solid) or out (dashed) of the room.

experimental room during each session. Each day $\mathrm{E}$ stayed in the room for at least $2 \mathrm{~min}$., which was usually long enough to record the latency of the first response. Beginning on day $32 \mathrm{E}$ was either in the room during the whole session or out for the last 28 min. and the arrangement of this variable was as follows: in, out, in, out, out, in, out, in, out, out, out, in, in, out, in, in, in, out. The Mann-Whitney U test was used to test the significance of the difference in performance between the "in" and "out" days.

\section{Results}

The number of presses during the nine "in" days $(\Sigma=214)$ is significantly greater $(p=.001)$ than during the nine "out" days $(\Sigma=57)$. The curves for the "in" and "out" days are presented in Fig. 1.

\section{Diseussion}

The results show that there is a significant effect of human presence on the extinction pressing rate of this cat. The fact that this effect was present during the last 9 sessions, when ACTH was not being injected, indicates that this phenomenon is not drug dependent. 
A large number of variables can be seen as possibly related to this result and will need to be investigated before the phenomenon is understood. Some of these are the role of handling and shaping by $\mathrm{E}$, the role of specific visual, olfactory and auditory cues, the presence or absence of this effect with animals of other phylogenetic levels, the influence of type and degree of motivation and type of response, and the extent to which other apparently incidental stimuli can be demonstrated to have similar effects as that here attributed to the presence of the experimenter. It is also possible that this result will aid in explaining the failures of replication attempts when slight differences in apparatus or procedure are involved.

\section{Notes}

1. The author is grateful to R. A. Cleghorn and Sz. Donhoffer for suggestions on the presentation of this paper.

2. We are indebted to Nordic Biochemicals, Montreal, P. Q., for supplies of ACTH.

\section{Reply}

We have read with interest the comments of Dr. James G. Taylor on our report of effects of prolonged prism wearing (Psychon. Sci., 1964, 1, 199-200). Dr. Taylor objects to our description of the results in terms of alteration of stimulus dependencies, preferring an explanation in terms of "amount of behavioral adaptation to the distorted retinal input."

It seems that Dr. Taylor must agree with us that stimulus-response relations have changed. His argument then is that the important stimulus conditions involved in the change of relations are ones in which we have behaved. In the paper in question, we were describing in summary form the results of our studies and did not discuss in any detail possible mechanisms underlying these results. We think that Dr. Taylor's remarks are directed towards a proposed mechanism of adaptation and hence are not in disagreement with the description in the paper. In one instance where we did discuss the conditions responsible for adaptation we suggested that our results might be attributed to "less restrictive conditions of activity"' (p. 200). In this case we would seem to be in agreement with Dr. Taylor.

This is not to say that we consider behavior to be involved in all aspects of adaptation to prisms or at least behavior in the gross functional sense in which Dr. Taylor seems to use it. Adaptation to curvature can apparently occur without such behavior (Gibson, J. J. J. exp. Psychol., 1933, 16, 1-31). Adaptation to displacement can apparently occur without such behavior (Wallach, H., Kravitz, J. H., \& Lindauer, J. Amer. J. Psychol., 1963, 76, 568-578). It is difficult to envision why adaptation to the chromatic fringes produced by a prism should be dependent on behavior in any gross functional sense. Furthermore to consider the distortion which Dr. Taylor uses as a case in pointthe convergence of horizontal lines above and below eye level, we find that the subjects who wore the prisms for prolonged periods did adapt to the downward slant of lines above eye level. In particular they did adapt to the downward slant of the ceilings specifically mentioned by Dr. Taylor. Six out of six subjects questioned in a final taped interview upon removing the prisms after 42 days of continuous wearing described the ceiling of the room they were in as sloping down to one sidean after effect of the distortion produced by the prism.

It is an important empirical question to determine when, under what conditions, and what kind of behavior is necessary for subsequent adaptation. Much of our current work is directed towards this goal. Certainly the imaginative experiments outlined in Dr. Taylor's book point to the importance of behavior in certain circumstances. But in the recent literature on adaptation to prismatic distortion there appear to be many paradoxical data. For this reason we believe considerable care must be taken in defining the nature of adaptation. To this end we attempted to measure adaptation to each of the distortions introduced by the prisms in a variety of ways. Our general procedure was to attempt to isolate a particular stimulus factor from others that would be distorted by the prism. Thus when a subject was making judgments relative to that aspect of a stimulus, it would be impossible for any other stimulus factor to affect his judgment even unconsciously. Only in this way could we be sure which aspects of the situation were determining the response. Consequently for precise measurement we would argue in favor of the "artificial laboratory situation" rejected by Dr. Taylor.

Herbert L. Pick, Jr. University of Minnesota Smith College

(See page 366 for comment by J. G. Taylor) 